\title{
Prevalencia de micosis superficial en pacientes con lesiones sugestivas de dermatofitosis
}

\author{
Aveiga Maldonado Ilka Patricia ${ }^{1}$, Beatriz M. Maldonado Lira ${ }^{2}$. \\ ilka.aveiga1778@gmail.com ${ }^{1}$, beatriz.maldonado@pucese.edu.ec.com² \\ ORCID ${ }^{1}$ : https://orcid.org/0000-0002-1619-7392, ORCID²: https://orcid.org/0000-0003-0600-3467 \\ Universidad Católica del Ecuador, Sede Esmeraldas \\ Esmeraldas-Ecuador
}

Recibido (01/07/20), Aceptado (17/07/20)

\begin{abstract}
Resumen: Este trabajo consistió en identificar la prevalencia de micosis superficial en pacientes con lesiones sugestivas de dermatofitosis. Para ello se realizó un estudio cuantitativo de corte longitudinal, prospectivo con alcance documental y exploratorio en el que participaron 42 pacientes que durante los meses del estudio acudieron con presunto diagnóstico de micosis superficiales; los resultados obtenidos demostraron que el 71,4\% de los pacientes son hombres, la mayoría con edad de 31 a 40 años, trabajan como obreros y tienen frecuente contacto con tierra. Los hongos encontrados fueron T. rubrum con 29\%, T. mentagrophytes con 7\%, Malassezia con $2 \%$ y en igual cantidad $2 \%$ en otras lesiones superficiales no causadas por hongos. Se pudo observar que los más afectados son hombres en edad reproductiva que tienen contacto con tierra $\mathrm{y}$ al estar expuestos al clima tropical y caluroso son propensos a adquirir micosis superficial.
\end{abstract}

Palabras Clave: Micosis superficial, hongos, lesiones sugestivas en piel, dermatofitosis.

\section{Prevalence of superficial mycosis in patients with lesions suggestive of dermatophytosis}

\begin{abstract}
This work consisted of identifying the prevalence of superficial mycosis in patients with lesions suggestive of dermatophytosis. To this end, a prospective, quantitative longitudinal-cut study was carried out with a documentary and exploratory scope in which 42 patients participated, who during the months of the study came with a presumed diagnosis of superficial mycosis; The results obtained showed that $71.4 \%$ of the patients are men, the majority aged between 31 and 40 years, work as laborers and have frequent contact with the ground. The fungi found were T. rubrum with $29 \%$, T. mentagrophytes with $7 \%$, Malassezia with $2 \%$ and in the same quantity $2 \%$ in other superficial lesions not caused by fungi. It was observed that the most affected are men of reproductive age who have contact with the ground and, being exposed to the hot tropical climate, are prone to acquiring superficial mycosis.
\end{abstract}

Keywords: Superficial mycosis, fungi, suggestive skin lesions, dermatophytosis.

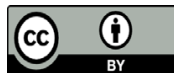




\section{I.INTRODUCCIÓN}

Este trabajo se centró en el estudio de las micosis superficiales, que son infecciones causadas por hongos, parásitos de la queratina. Estos pueden ser identificados a través del estudio macroscópico de las colonias y el estudio microscópico de sus estructuras en los cultivos; la micosis pueden ser profundas y de tipo superficial, esta última sucede cuando la infección se ubica en la parte más superficial de la piel y el cabello, es decir en tallos pilosos y células de la epidermis superficial, las personas que presentan este tipo de micosis son atendidas en áreas médicas de Medicina general y Dermatología por tratarse de afectaciones en la piel.

Las micosis superficiales se presentan de diferentes maneras de acuerdo al hongo que la produce, por lo que pueden aparecer como dermatofitosis, Candidiasis, Pitiriasis versicolor, este tipo de infecciones se dan especialmente en climas tropicales [1]. De acuerdo con Giusiana [1] las micosis superficiales son causadas por hongos llamados dermatofitos por lo que la infección que producen se les denomina dermatofitosis, aunque también se les conoce como tiñas o Tineas, los hongos que causan esta patología pertenecen a tres géneros: Trichophyton, Microsporum y Epidermophyton que afectan directamente a las uñas, piel, pelo y cuero cabelludo.

Para determinar si el paciente padece de dermatofitosis es necesario basarse en las características de las lesiones en el paciente y para confirmar un diagnóstico presuntivo se le realiza un análisis de laboratorio realizando un raspado cutáneo en el área afectada en preparados de hidróxido de potasio [2], además del examen directo para su confirmación. La muestra debe ser observada en microscopio y también se debe realizar un cultivo.

Entre los hongos filamentosos superiores se encuentran los dermatofitos que están conformados por los géneros Microsporum, Trichophyton y Epidermophyton que son causantes de micosis superficiales de la piel y sus anexos y son comúnmente conocidas como tiñas o Tineas [3].

De acuerdo con Aaron [2] la dermatofitosis es una infección producida por hongos parásitos de la queratina la cual está presente en la piel, pelo, cuero cabelludo y uñas, en esta última se les conoce como tiña ungueal u onicomicosis aunque la tiña es variada y se nombra de acuerdo a la parte del cuerpo que afecta, por ello hay tiña de barba, tiña del cuero cabelludo, además tiña corporal, tiña inguinal, tiña de los pies y la llamada reacción dermatofítide que es una inflamación provocada por la dermatofitosis y se localiza distante del sitio donde se localiza la micosis.

Las infecciones por hongos tienen una letalidad considerable puesto que de acuerdo al Fondo de Acción Mundial para las Infecciones por Hongos (GAFFI por sus siglas en inglés), alrededor de 300 millones de personas tienen alguna infección provocada por hongos cada año lo que más adelante termina con la pérdida de 1.5 millones de vidas [4].

En cuanto a la transmisión, los dermatofitos se clasifican en tres tipos dependiendo de donde habitan, por ello se conoce como Geofílicos los que habitan en la tierra y por este medio se transmiten a las personas, se consideran zoofílicos los que habitan en los animales y a través de ellos pasan la enfermedad y los antropofílicos son los que se contagian de persona a persona [5]. Por el tipo de contagio es fácil que las personas tiendan a adquirir una micosis superficial, especialmente los inmunodeprimidos (personas que tienen bajas las defensas).

El riesgo de adquirir la tiña es variado y se presentan por diferentes factores en las personas, entre los que se menciona la edad, puesto que los más susceptibles a adquirirla son los niños, además, el estar inmunocomprometidos por enfermedades metabólicas de base, tener enfermedades cutáneas como la ictiosis que es el engrosamiento y presencia de escamas en la piel, además el tener queratodermia que es el engrosamiento de las palmas de las manos y planta de los pies. Además son susceptibles las personas que son alérgicas y quienes utilizan ciertos tipos de medicamentos, como los esteroides. Los climas tropicales o subtropicales, son condicionantes para la tiña y puede aparecer luego de un golpe entre la uña y el dedo, a quienes sudan en manos y pies, por las condiciones del trabajo, el uso de piscinas y baños de uso general, compartir implementos y ropa contaminada al hacer ejercicios físicos y el no utilizar zapatos en lugares contaminados [6].

Es preciso mencionar que las personas con comorbilidades, es decir, quienes ya tienen una enfermedad como Virus de Inmunodeficiencia Humana (VIH), cáncer, diabetes y cualquier otra patología que pueda convertirlos en personas inmunodeprimidas, son más propensas a adquirir enfermedades producidas por hongos.

En este trabajo se identifica la prevalencia de micosis superficial en pacientes con lesiones sugestivas de dermatofitosis. El estudio se realizó en un Centro Médico privado durante el periodo marzo - mayo del año 2019, ubicado en la Provincia de Esmeraldas. 


\section{II.DESARROLLO}

La piel es el órgano más extenso del cuerpo humano cuya función principal es de proteger a las personas ante los cambios de temperaturas climáticas, de las lesiones e infecciones [7]. Existen varios microorganismos que pueden llegar a la piel para causar infecciones de manera invasiva y también superficial como lo hacen los dermatofitos que causan las micosis superficiales. Para [8], la aparición de las lesiones por hongos patógenos como los dermatofitos, posiblemente se remonta a la era mesozoica puesto que ellos consideran que en un principio las personas vivían en el suelo teniendo contacto con humanos y animales, de tal manera comenzaron las infecciones.

\section{A.Tipos de dermatofitos}

Los dermatofitos se presentan en diferentes tipos [9] de acuerdo con su hábitat natural como se muestra a continuación:

Antropofílicos: Se manifiestan principalmente en humanos y raras veces se transmiten a animales y tienen preferencias en algunas zonas del cuerpo humano dependiendo de los factores que favorezcan su incubación, estos podrían ser: clima, humedad excesiva, falta de higiene del hospedero, un calzado inadecuado, ropa ceñida, entre otros. Estos dermatofitos pueden contaminar el suelo de los gimnasios o de las piscinas donde existe humedad, toallas y atuendos deportivos del hombre, y de esta manera transferirse de una persona a otra.

Zoofílicos: Se encuentran en animales, y pueden transmitirse a humanos. En el suelo suelen tener una vida corta, sin embargo, en los pelos, plumas o escamas de los animales la vida de este hongo es larga y luego se depositan en las ropas, utensilios y muebles de la casa. Por lo tanto, la falta de higiene y educación sanitaria favorece la transmisión de la infección.

Geofílicos: se encuentran en el suelo, y desde allí se asocian con pelo, plumas y pezuñas en descomposición infectando tanto a humanos como animales, transmitiéndose de un hospedero a otro directa o indirectamente por fómites.

\section{B.Clasificación de los dermatofitos}

Los dermatofitos de clasifican en Trichophyton, Microsporum y Epidermophyton que afectan directamente a las uñas, al cabello y a la piel [1].

Cruz y Carvajal [3] opinan que el género Epidermophyton tiene sólo una especie patógena, E. floccosum, que puede producir la tiña de la ingle (eccema marginado de Hebra, Tinea cruris) así como la onicomicosis.

Tinea unguium es una infección progresiva y crónica que se manifiesta en cualquier parte de la uña. Los agentes etiológicos más involucrados pertenecen al género Trichophyton, es un hongo antropofílico y se transmite de persona a personas a través de fómites especialmente por el uso de instrumentos que se usan para el corte e higiene de las uñas, se presenta comúnmente en uñas del pie de adultos y puede ser de origen primario o diseminación de una tiña del cuerpo [10].

\section{La Candidiasis}

Es una infección causada por levaduras del género Candida, tiene manifestaciones clínicas muy variables, de evolución aguda, subaguda, crónica o episódica. Este tipo de hongo puede causar lesiones cutáneas, mucocutáneas, profundas o diseminadas esta enfermedad de la piel es de enorme importancia en la patología humana. Por su etiología es posible afirmar que puede penetrar en los tejidos y diseminarse por vía hemática provocando importantes reacciones severas en el organismo, pudiendo ocasionar sepsis y lesiones viscerales, por sus características bioquímicas y morfológicas se diferencian entre ellas, Candida albicans es la más conocida, además existen otras especies como C. tropicalis, C. krusei, C. parapsilosis, C. glabrata, C. dubliniensis, C. auris entre otras [11].

Un estudio realizado [7] en el que participaron 4257 pacientes a los que se les hizo un raspado en lesiones de piel, pelos, cuero cabelludo y uñas, se obtuvo que la mayoría (53,2\%) de participantes fueron mujeres y 46,8\% fueron hombres, las micosis presentadas fueron 44,7\% dermatofitosis; $28,4 \%$ candidosis; $22,4 \%$ Pitiriasis versicolor; $3,9 \%$ Onicomicosis por mohos, $0,5 \%$ Onicomicosis por Trichosporon y $0,1 \%$ por agentes acusantes de Tiña negra; mientras que la presencia de dermatofitosis se presentaron por hongos T. mentagrophytes $46,7 \%$, M. canis $25,1 \%$, T. rubrum $22,1 \%$, M. gypseum 4,2\% y E. floccosum $1,9 \%$.

Algunos autores [12], desarrollaron su investigación mediante la aplicación de un estudio descriptivo a 255 personas que fueron $57,3 \%$ mujeres y $42,7 \%$ hombres, a quienes les tomaron muestras de raspado primero con examen directo con $\mathrm{KOH}$ al $10 \%$ en una placa porta objetos y luego se realizó la observación microscópica con 
objetivo de 10x y 40x, finalmente se realizó el sembrado de las muestras, los resultados arrojaron que los hongos levaduriformes hallados con más frecuencia fueron: Candida spp. 53,3\%, Trichosporon spp 12,09\%, los dermatofitos fueron Trichophyton mentagrophytes 56,8\%, Trichophyton rubrum 32,4\%.

La investigación realizada por Aguinaga [13] fue desarrollada en la ciudad de Quito en Ecuador, realizando un estudio en una población de 3017 muestras de cultivos micológicos, como resultados se obtuvo que la mayor cantidad de participantes fueron personas adultas 49,804, de adultos mayores 45,01\%, adolescentes $2,22 \%$, niños $1,96 \%$ y lactantes $1 \%$, en cuanto a la presencia de hongos se encontraron hongos oportunistas Candida sp. 67,91\%, además Aspergillus 7,46\%, Cryptococcus neoformans 5,97\%, Histoplasma sp. con el 4,85\%, además Cladosporium sp. en $4,48 \%$ y otras menos de $4 \%$; de hongos no oportunistas estuvieron pequeños porcentajes de Microsporum sp. 0,18\%, Trichophyton sp. 3,27\% y en menor cantidad Epidermophyton floccosum 0,04\%.

En Perú, [14] se desarrolló una investigación con una muestra conformada de 83 niños a quienes les aplicó una encuesta y además se les recolectó muestra mediante raspado en la piel y luego realizó un examen directo colocando la muestra en una lámina porta objetos con $\mathrm{KOH}$ al 10\%, como resultados obtuvo que el 50,6\% fueron mujeres y 49,40\% fueron hombres además que el $51.8 \%$ presentó hongos filamentosos de tipo Dermatofito; $14.5 \%$ presentó levaduras del género Cándida sp., 3.6\% de tipo Malassezia sp y 30.1\% dio como resultado negativo.

El estudio realizado por [15] utilizó una muestra de 464 pacientes, obtuvo como resultado que el 72\% fueron mujeres, el mayor grupo etario fue el 70,1\% entre 31 y 65 años, $76 \%$ del área urbana, con el 41\% con ocupación en quehaceres domésticos; en cuanto a los hongos encontrados halló en uñas de las manos 38\% Candida sp., Candida parapsilosis 32\%, Trichophyton rubrum 11\%, y en menor cantidad Candida albicans $4 \%$, C. tropicalis 3,5\% e igual porcentaje de mixtas; en cuanto a las uñas de los pies encontró la mayor cantidad en Trichophyton rubrum 56\%, Candida sp. 10\%, Trichophyton mentagrophytes, C. tropicalis y Fusarium sp. se presentaron en el 6\%.

\section{III.METODOLOGÍA}

Para el desarrollo de esta investigación se hizo un estudio cuantitativo porque se realizó una valoración numérica porcentual de los resultados, fue prospectivo porque no se tomó información recopilada ni analizada en el pasado, sino que fue recolectada durante el desarrollo de esta investigación. Además, es de tipo documental porque se analizaron otros estudios y se revisó la información bibliográfica existente. También es posible mencionar que este trabajo tiene un alcance exploratorio porque se examinó una situación poco estudiada en el que se manipuló artificialmente el objeto de estudio o variable para ver el comportamiento del mismo y determinar sus características, además este estudio fue de corte longitudinal en vista que los resultados no se obtuvieron en un solo tiempo sino al pasar de los días viendo cómo se comportaba el objeto estudiado desde que fue manipulado.

En las variables se hallaron una dependiente que fueron las Micosis superficiales y dos variables independientes que fueron la sociodemográfica y los dermatofitos.

Micosis superficiales: Las micosis superficiales son enfermedades de tipo infecciosas que son causadas por hongos queratinofílicos llamados dermatofitos por lo que la infección que producen se les denomina dermatofitosis, aunque también se los conoce como tiñas o Tineas [1].

Socio demografía: La socio demografía es la que se encarga del análisis e interpretación de la demografía también analiza e interpreta los datos como edad, etnia, matrimonios, muertes, entre otros, mide la proyección de los datos obtenidos [16].

Dermatofitosis: Las dermatofitosis son infecciones producidas por hongos parásitos de la queratina presente en la piel, pelo y uñas, en esta última se las conoce como tiña ungueal u onicomicosis [2].

La población estuvo determinada por 42 personas voluntarias, se consideraron los siguientes criterios de inclusión y exclusión:

Los criterios de inclusión y exclusión fueron los siguientes:

A. Criterios de inclusión

- Pacientes que presentaron lesiones sugestivas en la piel, pelo y uñas compatibles con dermatofitosis, sin distingo de edad ni sexo.

-Se incluyeron a aquellos pacientes que aceptaron voluntariamente participar en el estudio.

- Pacientes que firmaron la hoja se consentimiento informado. 
B. Como criterio de exclusión

- Fueron excluidos aquellos pacientes que presentaron otras lesiones como psoriasis, eccema, dermatitis, escabiosis no compatibles con micosis.

-Se excluyeron las muestras que se contaminaron puesto que no permitirían obtener un resultado claro.

-Además no fueron incluidos los pacientes que no facilitaron los datos completos para el llenado de la ficha de datos, puesto que la información quedaría incompleta para su tabulación.

\section{IV.RESULTADOS}

Además de los 42 pacientes con lesiones en la piel fueron separados por grupo etario obteniendo que $28.6 \%$ de ellos estaban en edades comprendidas entre los 31 y 40 años; 23,8\% con edad entre 51-60 años, mientras que $16,7 \%$ fueron $41-50$ años de edad, $11,9 \%$ tenían entre $61-70$ años, otro $11,9 \%$ eran personas mayores de 70 años y en menor porcentaje fueron $7,1 \%$ que eran menores de 20 años; cabe señalar que no se presentaron pacientes en edades entre 21 y 30 años.

En referencia al género de los pacientes con lesiones sugestivas de dermatofitosis en el Centro Médico privado, que conformó la muestra de este estudio, se obtuvo que de los 42 participantes la mayoría que representan el 71,4\% fueron de sexo masculino mientras que el $28,6 \%$ fueron de sexo femenino.

Sobre las actividades laborales desarrolladas por los participantes, se obtuvo que el mayor porcentaje $45,2 \%$ son del sector laboral obrero, en menor cantidad representada por el 35,7\% trabajan en la administración del hogar, mientras que $7,1 \%$ son estudiantes, igualmente otro $7,1 \%$ pertenecen al sector agropecuario, finalmente en menor porcentaje se obtuvo que apenes un $4,8 \%$ tienen relaciones laborales de dependencia, es decir, son empleados.

En referencia al estado de los pacientes antes de la toma de muestra, los pacientes con lesiones sugestivas de dermatofitosis en el Centro Médico privado de Esmeraldas indicaron que habían recibido tratamiento médico antes de la realización de los análisis de este estudio, se obtuvo como respuesta que más de la mitad de ellos 59.5\% se habían aplicado tratamiento previo al análisis de laboratorio y en menor cantidad que fue el $40.5 \%$ no se habían medicado.

Para determinar las fuentes de contactos frecuentes a los que están expuestos los pacientes con lesiones sugestivas de dermatofitosis en el Centro Médico, entre las opciones tierra, animales o plantas, la mayor parte que fue el $70,0 \%$ respondieron tener contacto frecuente con el suelo específicamente con tierra, mientras que el 19,5\% indicó mantener contacto directo con animales y en menor cantidad $9,5 \%$ respondieron que tienen contacto directo con las plantas.

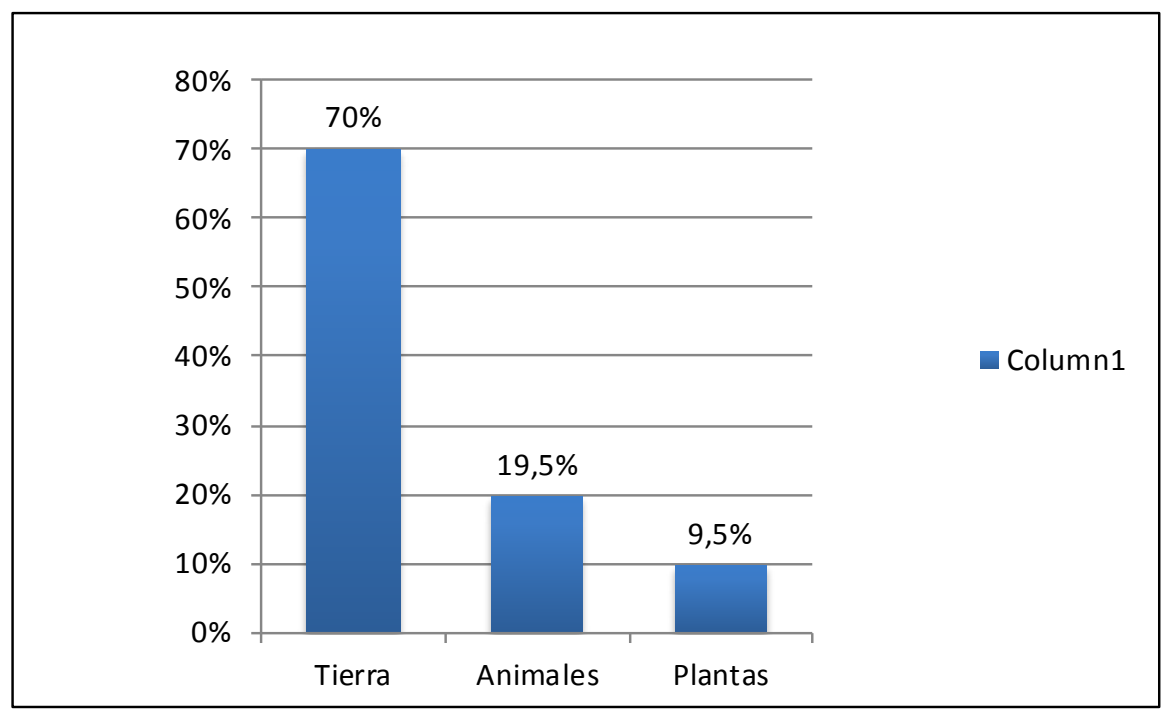

Figura 1. Fuentes de contacto.

Es preciso recalcar que el $60 \%$ de las muestras resultaron contaminadas, por lo que los análisis se desarrollaron en el 40\% restante. Luego de los procedimientos realizados (figura 2) se evidenció el agente etiológico aislado 
con mayor frecuencia y fue T. Rubrum que estuvo presente en un $29 \%$ de los pacientes estudiados, seguido de T. mentagrophytes en un porcentaje mucho más bajo siendo el $7 \%$ de los pacientes y en menor cantidad fueron de Malassezia sp. en un 2\% de igual manera 2\% de otras lesiones superficiales encontradas que no fueron causadas por hongos.

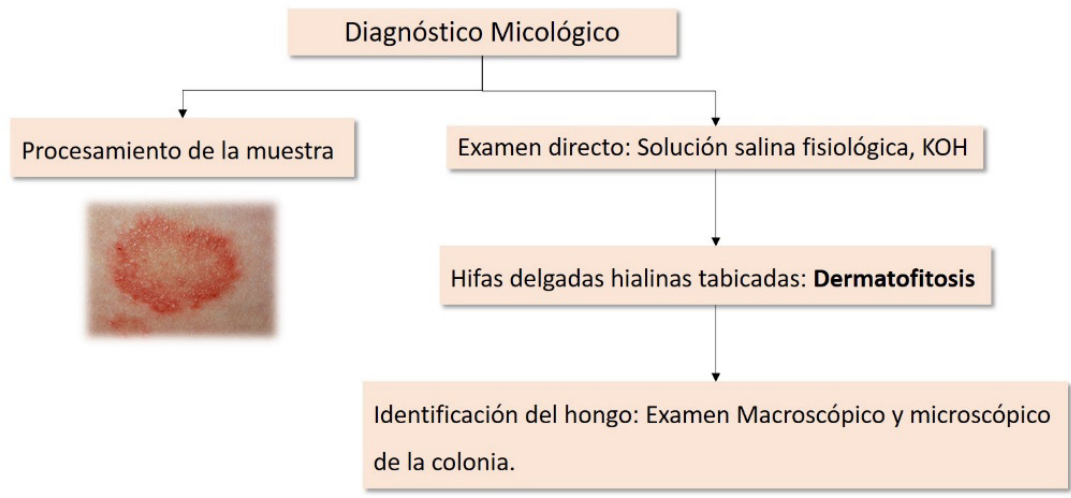

Figura 2. Diagnóstico micológico

Con los resultados obtenidos que fueron anteriormente presentados se evidenció que la mayor cantidad de pacientes fueron de sexo masculino lo que representa el 71,4\%, mientras que el 28,6\% fueron mujeres; lo que no coincide con el estudio de [7], [12], [14] ni con el de [15] puesto que ellos obtuvieron que la mayor cantidad sus participantes fueron de sexo femenino, esto indica que los hombres en Esmeraldas son más propensos de adquirir micosis superficiales y en mucho menor proporción las mujeres.

En referencia al grupo etario, la mayoría en este estudio fueron adultos jóvenes entre 31 y 40 años seguidos de personas de 51 a 60 años, lo que se asemeja a los resultados de [13], [15] quienes también obtuvieron la mayor cantidad de pacientes en edad adulta; esto indica que la micosis superficial puede afectar a personas adultas en edad productiva.

En cuanto a los hongos encontrados en este estudio, fueron Trichophyton rubrum, Trichophyton mentagrophytes y en menor cantidad Malassezia sp. Estos eran supuestos por algunos autores [17] mientras que los dos primeros también fueron hallados por [7], [12], [15], [18] y [19] se coincide con [14] quien también encontró Malassezia sp; en cambio los resultados de hongos encontrados en este estudio no coinciden con ninguno de los hallados por [13]. Estos resultados señalan que Trichophyton rubrum, Trichophyton mentagrophytes son los que más frecuentemente se observan como agentes causales de micosis superficiales.

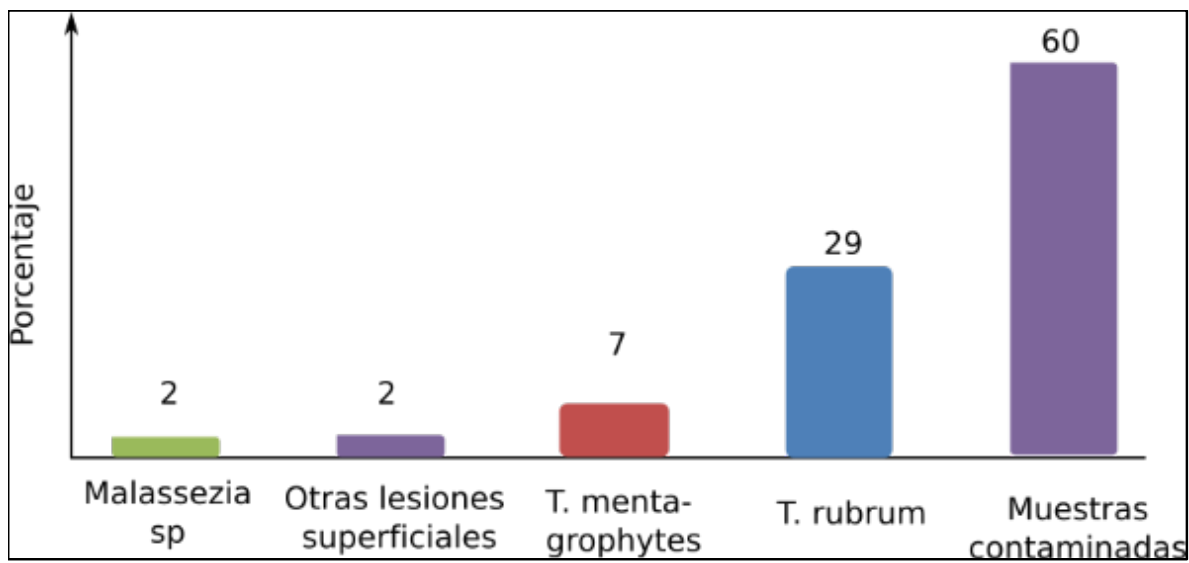

Figura 3. Resultado de los Cultivos por etiología, de pacientes investigados en el Centro Médico Privado, Esmeraldas, período de marzo a mayo del año 2019. 


\section{V.CONCLUSIONES}

Luego de haber completado el estudio propuesto, se ha llegado a la conclusión que las micosis superficiales pueden afectar sobre todo a las personas en edad productiva puesto que se evidenció que la mayoría de los pacientes que participaron en este y todos los estudios presentados fueron adultos jóvenes; en referencia al género, no se coincidió con ningún otro estudio, lo que evidencia que en Esmeraldas los hombres son más propensos que las mujeres para adquirir esta enfermedad.

Después de conocer que los pacientes con lesiones sugestivas de dermatofitosis que fueron atendidos en el Centro Médico durante el periodo de estudio realizan sus funciones fuera de casa y la mayoría trabaja con constante contacto con la tierra; se ha llegado a la conclusión que éste es uno de los factores predisponentes para que contraigan las micosis superficiales, puesto que el realizar las actividades laborales fuera de casa, con constante contacto con tierra, expuestos al calor en una ciudad tropical y el clima húmedo como Esmeraldas permite que los pacientes sean más vulnerables y pueden crear en el hospedero las condiciones idóneas para la aparición de lesiones sugestivas causada por hongos; cabe mencionar que manifestaciones de estas lesiones van a variar dependiendo del estado de inmunidad, edad, estilo de vida y educación higiénica que tenga el hospedero, dado que estas micosis se van incrementando por el exceso de sudoración o mal secado de los pies y de las manos.

Este estudio reveló que los hongos que más afectan la salud de los pacientes con lesiones sugestivas de dermatofitosis que fueron atendidos en el Centro Médico son de la variedad de Trichophyton rubrum, Trichophyton mentagrophytes y en menor cantidad fueron de Malassezia sp. que precisan de humedad, el calor, y la falta de higiene personal en los hospederos para que suceda el contagio, por ello se denota su presencia y prevalencia sobre todo de Trichophyton rubrum, y Trichophyton mentagrophytes que se encontraron en una cantidad considerablemente elevada.

El estudio de las enfermedades causadas por hongos patógenos y contaminantes puede marcar una diferencia en la terapéutica antifúngica, no basta con conocer los géneros y especies de las infecciones que afectan el tejido subcutáneo, también es importante establecer su relación con los hábitos de higiene del paciente y la región anatómica afectada.

Al estudiar las micosis superficiales hay que considerar que no basta con aplicar principios científicos, sino apuntar a un tratamiento antifúngico acertado para evitar fracasos terapéuticos que conlleve a la resistencia a estos. Para ello es imperante realizar una adecuada toma de muestra del sitio activo de la lesión y explicar a los pacientes la importancia de no colocar ningún tipo de medicamentos tópico o sustancias antes de la toma de la muestra, realizar una adecuada y completa historia micológica que permitirá conocer los antecedentes que han tenido los pacientes con una posible fuente de infección, bien sean animales, el compartir uso de fomites y conocer sus hábitos de higiene personal.

Por lo anteriormente expuesto es importante, educar a la población sobre cómo altera los resultados de análisis micológicos al colocarse medicación y remedios caseros sobre las lesiones sin prescripción médica y el revés que pudiera aparecer como consecuencia de automedicarse. El paciente debe acudir al médico apenas observe cambios en uñas, pliegues interdigitales, sea esta por descamaciones o despigmentación de la piel y cuero cabelludo. En esta misma campaña educativa dirigida al paciente, aclarar la importancia del uso de calzado que den una adecuada ventilación para así evitar el hospedaje de microorganismos oportunistas que terminen afectando la calidad de vida del paciente y su salud en general.

\section{RECONOCIMIENTO}

Reconocemos el aporte brindado por la Pontificia Universidad Católica del Ecuador, Sede Esmeraldas, por su apoyo en la elaboración de este trabajo.

\section{REFERENCIAS}

[1]K. Taco, «Frecuencia de hongos tinea unguium de los pies en aspirantes a policías por cultivo micológico en la escuela de formación Cbos.,» Universidad Central del Ecuador, Quito-Ecuador, 2015.

[2]D. Aaron, «Generalidades sobre la dermatofitosis.,» 2018. [En línea]. Available: https://www.msdmanuals.com/ es/professional/trastornos-dermatol $\% \mathrm{C} 3 \% \mathrm{~B} 3$ gicos/infecciones-mic $\% \mathrm{C} 3 \% \mathrm{~B} 3$ ticas-cut $\% \mathrm{C} 3 \% \mathrm{~A} 1$ neas/generalidades-sobre-la-dermatofitosis.

[3]R. Cruz, L. Carvajal, S. Pérez y V. Rodríguez, «Aislamiento de Microsporum spp. en dermatofitosis en pacientes de la región de Valparaíso - Chile.,» Revista Argentina de Dermatología, vol. 98, nº 1, pp. 27-37, 2017. 
[4]RDF, «Red de Infecciones Fúngicas de Guatemala.,»[En línea]. Available: http://fungired.gt/index.php/ que-es-fungired.

[5]C. d. Leal., «Micosis superficiales.,» [En línea]. Available: https://dermatologialeal.wordpress.com/enfermedades-indice/micosis-superficiales/.

[6]Instituto Mexicano de Seguro Social, «Diagnóstico y tratamiento de tiña y onicomicosis en el primer nivel.,» [En línea]. Available: http://www.imss.gob.mx/sites/all/statics/guiasclinicas/086GRR.pdf.

[7]D. Lemus-Espinoza, M. T. Maniscalchi, O. Villarroel, S. Bónoli, F. Wahab y O. García, «Micosis superficiales en pacientes del estado Anzoátegui, Venezuela, período 2002-2012.,» Investigación Clínica, vol. 55, nº 4, pp. 311 $320,2014$.

[8]M. Uribe y N. Cardona-Castro, «Mecanismos de adherencia e invasión de dermatofitos a la piel.,» CES Medicina, vol. 27, $\mathrm{n}^{\mathrm{o}}$ 1, pp. 67-75, 2013.

[9]G. Estrada y M. Ramírez-Galeano, «Micología General.»» 2019. [En línea]. Available: http://repositorio.ucm. edu.co:8080/jspui/handle/10839/2654.

[10]F. Gómez-Daza, «Caso clínico No 36 diagnóstico definitivo.,» 2009. [En línea]. Available: https://piel-1.org/ blog/7134.

[11]M. Biasoli, «Candidiasis.,» [En línea]. Available: https://www.fbioyf.unr.edu.ar/evirtual/file.php/118/MATERIALES_2013/TEORICOS_2013/CANDIDIASIS_2013-1.pdf.

[12]G. Estrada-Salazar y J. Chacón-Cardona, «Frecuencia de dermatomicosis y factores asociados en población vulnerable. Manizales, Colombia.,» Revista de Salud Pública, vol. 18, nº 6, pp. 953-962, 2016.

[13]M. Aguinaga, « Infecciones fúngicas oportunistas en pacientes del Hospital Carlos Andrade Marín en el periodo 2015-2017,» Universidad Central del Ecuador, Quito-Ecuador, 2018.

[14]K. Panta, «Prevalencia de micosis superficial en niños y adolescentes de 09-16 años, del Caserío el Papayo, Tambogrande, diciembre 2017- abril 2018.,» Universidad de San Pedro, Sullana-Perú, 2018.

[15]L. Insfrán, M. Meza, T. Aldama, O. Aldama, J. Pereira, A. Feliciano y J. García, «Características epidemiológicas de las onicomicosis en la consulta dermatológica.,» Revista del Nacional (Itauguá), vol. 11, nº 2, pp. 2-8, 2019. [16]ECURED, «Demografía.,» [En línea]. Available: https://www.ecured.cu/Demograf\%C3\%ADa.

[17]S. España y E. T., «Situación de la micosis superficial en Ecuador,» Universidad Católica Santiago de Guayaquil, Guayaquil-Ecuador, 2019.

[18]D. Zambrano y G. Chang, «Factores asociados con la aparición de dermatofitosis superficiales en pacientes de la Consulta externa del servicio de dermatología del Hospital Naval durante el año 2017,» Universidad Católica Santiago de Guayaquil, Guayaquil-Ecuador, 2019.

[19]C. López, D. Morillo y P. Plaza, «Estudio Trasversal: Micosis Superficiales en Niños Escolares de una Parroquia Rural de Cuenca, Ecuador.,» Revista Médica HJCA, vol. 9, nº 3, pp. 249 - 254, 2017.

\section{RESUMEN CURRICULAR}

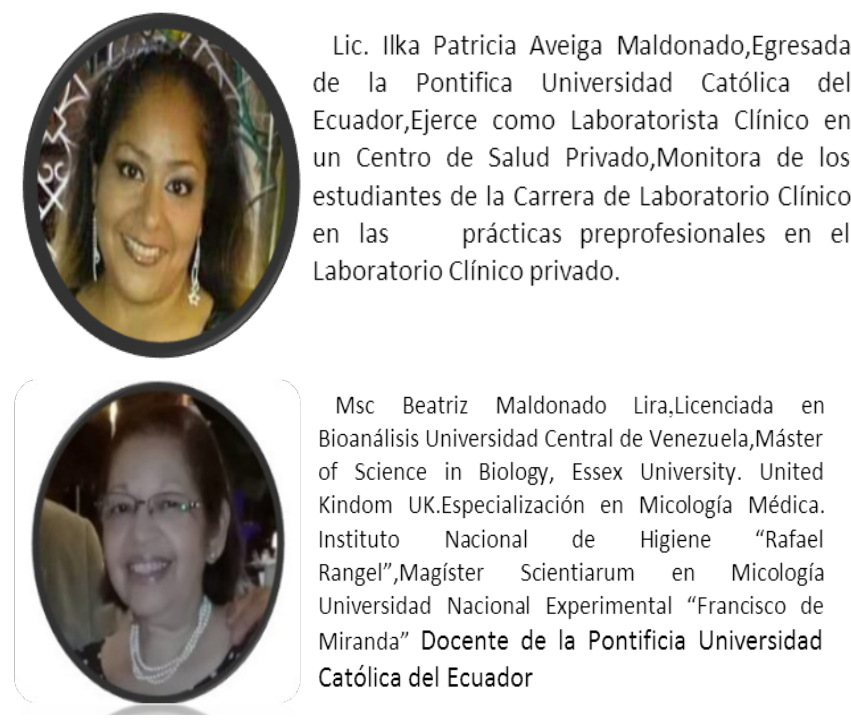

